\title{
Detection of Blood Traces in Human Pericardial Fluid Using Microwaves
}

\section{Anil Lonappan}

Department of Electrical Engineering, Mangosuthu University of Technology, Durban, South Africa.

Email: lonappan@mut.ac.za

Received August 12 ${ }^{\text {th }}, 2010$; revised September 1 $1^{\text {st }}, 2010$; accepted September $25^{\text {th }}, 2010$.

\begin{abstract}
This communication reports a novel method of analyzing pericardial fluid and finding blood traces present based on the measurement of the dielectric properties at microwave frequencies. The experiment was performed by cavity perturbation method in the S-band of microwave frequency with the pericardial fluid from healthy persons as well as from patients suffering from pericardial bleeding. It is noted that considerable variation in the dielectric properties of patient samples with the normal healthy samples and these measurements were in good agreement with clinical analysis. This measurement technique and the method of extraction of pericardial fluid are simple and both are non invasive. These results give light to an alternative in-vitro method of diagnosing onset pericardial bleeding abnormalities using microwaves without surgical procedure.
\end{abstract}

Keywords: Pericardial Fluid, Pericardial Bleeding, Cavity Perturbation, Clinical Analysis

\section{Introduction}

The survival rate is increased in heart patients based on the earlier detection of heart abnormalities or rhythm. Numerous diagnostic techniques are available for detecting heart abnormalities and most of the techniques are either invasive or noninvasive and most of them are time consuming procedures. These concerns augment the search for new techniques that can find other physical tissue properties at the onset of heart abnormalities.

Pericardial fluid is secreted by the serous membrane on the pericardious sac on the outside of the heart. The pericardial cavity contains between 15 and $50 \mathrm{ml}$ of pericardial fluid. It is similar to the serous fluid that is found in the brain for cushioning and ability to move semifreely. In pericardial bleeding there is the presence of pericardial fluid with blood and is an abnormal condition and there will be accumulation of fluid in the pericardial cavity. Because of the limited amount of space in the pericardial cavity, this fluid accumulation will lead to an increased intrapericardial pressure and can negatively affect heart function. When there is a pericardial bleeding with enough pressure to adversely affect heart function, this is called cardiac tamponade. While small bleeding occur in many clinical scenarios and are not necessarily dangerous, large and rapidly accumulating bleeding may cause cardiac tamponade, a life-threatening complication.
Ben-Horin et al. studied the composition of pericardial fluid in patients undergoing open heart surgery [1]. Diagnosing the onset pericardial bleeding abnormalities are crucial to increase the survival rate and the present study reports a new method of detecting the onset of pericardial bleeding based on the measurement of the dielectric properties of pericardial fluid using cavity perturbation technique in the S-band of microwave frequencies.

Medical science has advanced greatly in the last century, there is still much that is not understood about the way the human body interacts with many things, including microwaves. The potential for medical uses of microwaves lies in two areas therapeutic and diagnostic field and many new medical microwave devices are also developed [2,3]. All the known therapeutic uses of microwaves involve the heating of tissue. Carefully controlled, microwave-generated heat can have a therapeutic effect on a number of ailments. Microwave medical devices ability depends upon how far microwaves can deeply penetrate into living tissues. The depth to which microwaves can penetrate tissues is primarily a function of the dielectric properties of tissues and of the frequency of the microwaves. Lower the water content of the tissue the deeper a wave at a given frequency and lower the frequency the deeper is the depth of penetration into tissues with given water content. Thus, there is a need to 
study the interaction of microwave with tissues especially its effect on biological materials. The key element in the microwave study is the determination of the absorbed energy. The amount of energy absorbed is a function of the complex permittivity of a material [4]. Hence, it is crucial to know the dielectric properties of biological materials and the various constituents thereof. Exhaustive studies of dielectric parameters of various human tissues and body fluids at different RF frequencies have been reported [5-7]. Different measurement techniques are used to measure the complex permittivity of a material and the chosen technique depends on various factors such as the nature of the sample and the frequency range used [8-11]. When only very small volumes of the sample are available, the cavity perturbation technique is an attractive option as it requires only minute volumes for the measurement [12]. This makes it suitable for the dielectric study of pericardial fluid, as only very small volumes can be only be extracted by procedure. The rectangular cavity perturbation technique has been employed for the measurement of the dielectric parameters of pericardial fluid samples obtained from healthy persons as well as from patients with pericardial bleeding in this work, in the frequency range 2 to $3 \mathrm{GHz}$. It is noticed that a remarkable change in the dielectric properties of bleeding samples with the normal healthy samples and these measurements were in good agreement with clinical analysis. This microwave measurement procedure is simple and extraction of pericardial fluid from persons is least painful and nonsurgical in nature. These results prove an alternative in-vitro method of detecting the onset of pericardial bleeding based on the measurement of the dielectric properties of pericardial fluid using microwaves without surgical procedure.

\section{Sample Preparation}

The skin of the chest will be cleaned with antibacterial soap and a small needle is inserted into the chest between the ribs into the pericardium, which is the thin sac that surrounds the heart, and a small amount of fluid is withdrawn. Then the samples were filled in the sample holder and kept at $1{ }^{\circ} \mathrm{C}$. Measurements were carried out on samples which were less than one day old.

\section{Material and Methods}

The experimental set-up consists of a transmission type S-band rectangular cavity resonator, HP 8714 ET network analyser. The cavity resonator is a transmission line with one or both ends closed. The numbers of resonant frequencies are determined by the length of the resonator. The resonator in this set-up is excited in the $\mathrm{TE}_{10 \rho}$ mode. The sample holder which is made of glass in the form of a capillary tube flared to a disk shaped bulb at the bottom is placed into the cavity through the non-radiating cavity slot, at broader side of the cavity which can facilitate the easy movement of the holder. The resonant frequency $f_{o}$ and the corresponding quality factor $Q_{o}$ of the cavity at each resonant peak with the empty sample holder placed at the maximum electric field are noted. The same holder filled with known amount of sample under study is again introduced into the cavity resonator through the nonradiating slot. The resonant frequency of the sample loaded cavity is selected and the position of the sample is adjusted for maximum perturbation (i.e. maximum shift of resonant frequency with minimum amplitude for the peak). The new resonant frequency $f_{s}$ and the quality factor $Q_{s}$ are noted. The same procedure is repeated for other resonant frequencies.

\section{Theory}

When a material is introduced into a resonant cavity, the cavity field distribution and resonant frequency are changed which depend on shape, electromagnetic properties and its position in the fields of the cavity. Dielectric material interacts only with electric field in the cavity

According to the theory of cavity perturbation, the complex frequency shift is related as [12]

$$
\begin{gathered}
-\frac{d \Omega}{\Omega} \approx \frac{\left(\bar{\varepsilon}_{r}-1\right) \int_{V_{S}} E \cdot E_{0}^{*} d V}{2 \int_{V_{c}}\left|E_{0}\right|^{2} d V} \\
\operatorname{But} \frac{d \Omega}{\Omega} \approx \frac{d \omega}{\omega}+\frac{j}{2}\left[\frac{1}{Q_{S}}-\frac{1}{Q_{0}}\right]
\end{gathered}
$$

Equation (1) and (2) and separating real and imaginary parts results

$$
\begin{aligned}
& \varepsilon_{r}^{\prime}-1=\frac{f_{o}-f_{s}}{2 f_{s}}\left(\frac{V_{c}}{V_{s}}\right) \\
& \varepsilon_{r}^{\prime \prime}=\frac{V_{c}}{4 V_{s}}\left(\frac{Q_{o}-Q_{s}}{Q_{o} Q_{s}}\right)
\end{aligned}
$$

Here, $\overline{\varepsilon_{r}}=\varepsilon_{r}^{\prime}-j \varepsilon_{r}^{/ /}, \overline{\varepsilon_{r}}$ is the relative complex permittivity of the sample, $\varepsilon_{\mathrm{r}}^{\prime}$ is the real part of the relative complex permittivity, which is known as dielectric constant. $\varepsilon_{\mathrm{r}}^{/ /}$is the imaginary part of the relative complex permittivity associated with the dielectric loss of the material. $\mathrm{V}_{\mathrm{s}}$ and $\mathrm{V}_{\mathrm{c}}$ are corresponding volumes of the sample and the cavity resonator. The conductivity can be related to the imaginary part of the complex dielectric constant as: 


$$
\sigma_{e}=\omega \varepsilon^{\prime \prime}=2 \pi f \varepsilon_{0} \varepsilon_{r}^{\prime \prime}
$$

\section{Results and Discussions}

The microwave studies of pericardial fluid samples were done using cavity perturbation technique collected from healthy donors as well as from the patients and the results are shown in Figures 1,2. These results are novel using microwave techniques for the case of pericardial fluid. Clinical evaluation of the pericardial fluid samples is also done and the results are tabulated in Table $\mathbf{1}$. They found it was relatively rich in lactate dehydrogenase, low in protein and high in lymphocytes and monocytes. From Figure 1 it is noticed that the bleeeding samples exhibit a higher dielectric constant than that of the normal samples. In Figure 2 the variation of conductivity of normal and bleeding samples are plotted. It can be found that distinct variation in the conductivities of normal samples and the bleeding samples. The increase in conductivity in bleeding samples is due to the presence of higher level of protein contents such as al-

Table 1. Variation of constituents in normal pericardial fluid samples as well as pericardial bleeding samples.

\begin{tabular}{|c|c|c|c|c|c|c|}
\hline \multirow[b]{2}{*}{ Sample } & \multirow[b]{2}{*}{ Colour } & \multirow{2}{*}{$\begin{array}{c}\text { Lactate } \\
\text { Dehydro- } \\
\text { genase } \\
\left(\mathbf{1 0}^{3} / \mathbf{m m}^{3}\right)\end{array}$} & \multirow{2}{*}{$\begin{array}{l}\text { Lypho- } \\
\text { cytes }\left(10^{3} /\right. \\
\left.\mathrm{mm}^{3}\right)\end{array}$} & \multirow{2}{*}{$\begin{array}{l}\text { Mocytes } \\
\left(10^{3} / \mathbf{m m}^{3}\right)\end{array}$} & \multicolumn{2}{|c|}{ Proteins(gm/dL) } \\
\hline & & & & & Albumin & $\begin{array}{c}\text { Globu- } \\
\text { lin }\end{array}$ \\
\hline PN-1 & Clear & 2.2 & 0.5 & 1.4 & 3.5 & 3.4 \\
\hline PN-2 & Clear & 1.9 & 0.4 & 1.2 & 4.2 & 2.9 \\
\hline $\mathrm{PN}-3$ & Cloudy & 2.6 & 0.6 & 1.5 & 3.8 & 3.1 \\
\hline $\mathrm{PN}-4$ & Clear & 2.1 & 0.5 & 1.3 & 4.9 & 2.3 \\
\hline PE-5 & Bloody & 2.2 & 1.8 & 4.3 & 13.9 & 14.1 \\
\hline PE-6 & Bloody & 2.1 & 1.9 & 4.0 & 12.8 & 10.3 \\
\hline PE-7 & Bloody & 2.7 & 1.3 & 3.9 & 16.2 & 13.4 \\
\hline PE-8 & Bloody & 3.1 & 1.4 & 4.2 & 14.4 & 15.6 \\
\hline
\end{tabular}

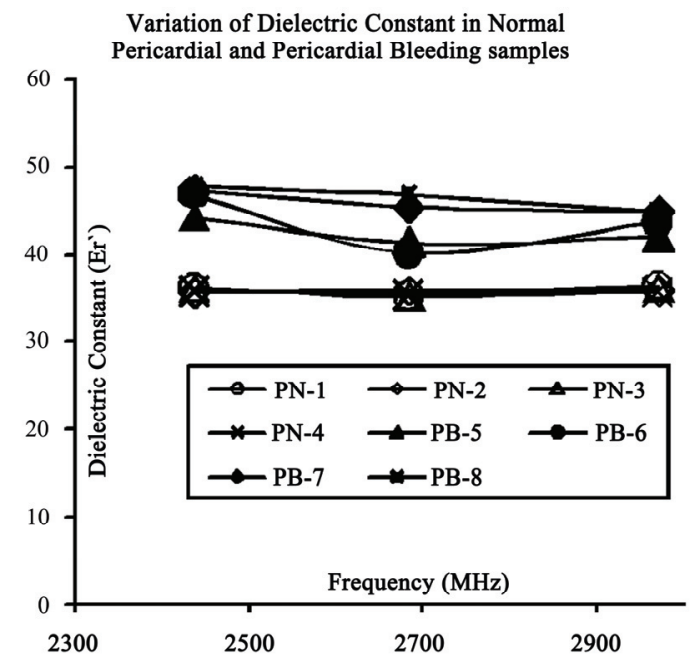

Figure 1. Variation of dielectric constant in normal pericardial sample and pericardial bleeding samples.

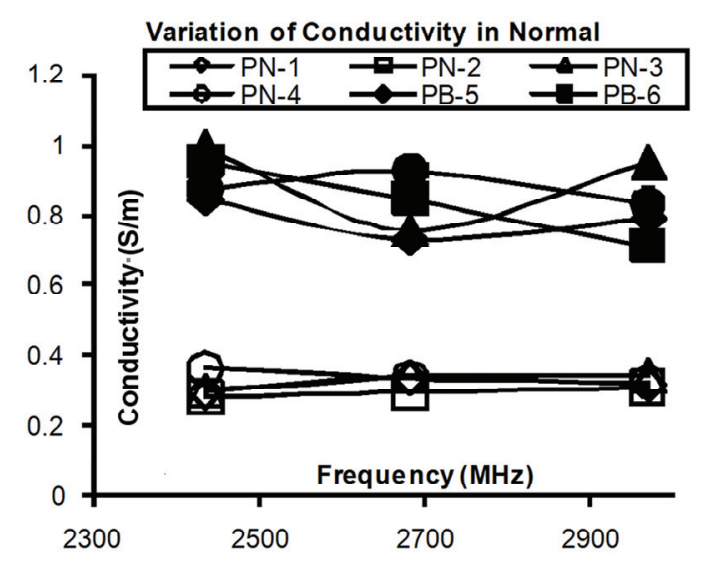

Figure 2. Variation of conductivities in normal pericardial sample and pericardial bleeding samples.

bumin and globulin as well as blood traces. Thus in the specified band of frequencies, normal pericardial and pericardial bleeding samples were studied and exhibit distinct variation of dielectric constant and conductivity with frequency.

\section{Conclusions}

The microwave characterisation of the pericardial samples is done using cavity perturbation technique. The cavity perturbation technique is quick, simple, and accurate and it requires very low volume of sample for measuring the dielectric properties of tissue samples like pericardial samples. It is observed that a remarkable change in the dielectric properties of pericardial bleeding samples with the normal healthy samples and these measurements were in good agreement with clinical analysis. This measurement technique is simple and the extraction of pericardial fluid from persons is least painful and nonsurgical in nature. These results prove an alternative in-vitro method of diagnosing onset pericardial bleeding abnormalities using microwaves without surgical procedure.

\section{REFERENCES}

[1] S. Ben Horin, A. Shinfeld, E. Kachel, A. Chetrit and A. Livneh, "The Composition of Normal Pericardial Fluid and Its Implications for Diagnosing Pericardial Effusions," American Journal of Medicine, Vol. 118, No. 6, 2005, pp. 636-640.

[2] A. Rosen and H. D. Rosen, "New Frontiers in Medical Device Technology," Wiley, New York, 1995.

[3] "Mini-Special Issue on RF/Microwave Applications in Medicine," IEEE Transactions on Microwave Theory Technique, Vol. 48, 2000.

[4] A. Von Hippel, "Dielectric and Waves," Artech House, 1995.

[5] S. Gabriel, R. W. Lau and C. Gabriel, "The Dielectric 
Properties of Biological Tissues: II. Measurements on the Frequency Range $10 \mathrm{~Hz}$ to $20 \mathrm{GHz}$," Literature Survey, Physics Medicine Biology, Vol. 41, No. 11, 1996, pp. 22512269.

[6] H. F. Cook, "Dielectric Behavior of Human Blood at Microwave Frequencies," Nature, Vol. 168, 1951, pp 247-248.

[7] H. F. Cook, "The Dielectric Behavior of Some Types of Human Tissues at Microwave Frequencies," British Journal of Applied Physics, Vol. 2, 1951, pp 295-300.

[8] D. K. Ghodgaonkar, V. V. Varadan and V. K. Varadan, "Free Space Measurement of Complex Permittivity and Complex Permeability of Magnetic Materials at MicroWave Frequencies," IEEE Transactions on Instrumentation and Measurement, Vol. 19, 1990, pp. 387-394.

[9] D. K Ghodgaonkar, V. V. Varadan and V. K. Varadan, "A Free Space Method for Measurement ff Dielectric
Constant and Loss Tangents at Microwave Frequencies," IEEE Transaction on Instrumentation and Measurement, Vol. 38, 1989, pp. 789-793.

[10] W. Barry, "A Broadband, Automated, Stripline Technique for the Simultaneous Measurement of Complex Permittivity and Complex Permeability," IEEE Transaction on Microwave Theory and Techniques, Vol. 34, 1986, pp. $80-84$.

[11] Z. Abbas, R. D. Pollard and R. W. Kelsall, "A Rectangular Dielectric Waveguide Technique for Determination of Permittivity of Materials at W-Band," IEEE Transactions on Microwave Theory and Techniques, Vol. 46, 1998, pp 2011-2015.

[12] K. T. Mathew, "Perturbation Theory," Encyclopedia of $R F$ and Microwave Engineering, Vol. 4, 2005, pp. 37253735 . 